\title{
The role of combination therapy in managing pulmonary arterial hypertension
}

\author{
Hossein-Ardeschir Ghofrani ${ }^{1}$ and Marc Humbert ${ }^{2}$
}

Affiliations: 'Dept of Internal Medicine, University Hospital Giessen and Marburg, Giessen, Germany. ${ }^{2}$ Service de Pneumologie, Université Paris-Sud, AP-HP, Hôpital Bicêtre, Inserm U999, Le Kremlin Bicêtre, France.

Correspondence: Hossein-Ardeschir Ghofrani, Dept of Internal Medicine, Medical Clinic II/V, University Hospital Giessen and Marburg, Klinikstrasse 33, Giessen 35392, Germany.

E-mail: ardeschir.ghofranidinnere.med.uni-giessen.de

ABSTRACT Pulmonary arterial hypertension (PAH) is a complex, progressive disease with several pathobiological mechanisms, including the endothelin, nitric oxide and prostacyclin pathways. Current treatments for $\mathrm{PAH}$ target one of these pathways and, in more severe cases or instances of disease worsening, may be combined with a view to target multiple pathways in parallel. Treatment combination is performed sequentially (as an intensification from initial monotherapy) or upfront (use of two or more therapies in treatment-naïve patients). Whilst combination therapy has been historically considered to be an option for the treatment of $\mathrm{PAH}$, supporting evidence was typically limited to expert opinion, clinical experience and registry data.

Data from randomised controlled trials on sequential combination therapy in particular has grown in recent years, resulting in a change in the level of recommendations in the latest update to the PAH treatment algorithm. However, short-term trials have shown inconsistent results, and have not been powered to assess morbidity/mortality outcomes. More recent data from long-term trials suggest a potential clinical benefit associated with sequential combination therapy.

In this review we will introduce the concept of combination therapy, consider the latest evidence for both sequential and upfront combination therapy, and discuss additional considerations when initiating combination therapy in clinical practice.

-

@ERSpublications

Combination therapy in PAH management: concepts, evidence and potential considerations http://ow.ly/CEMld

\section{Introduction}

Pulmonary vasoconstriction and associated remodelling of the vascular walls are thought to be involved in the initiation of pulmonary arterial hypertension $(\mathrm{PAH})$, a pulmonary vascular disease that, if left untreated, can lead to heart failure and death $[1,2]$. The pathogenesis of PAH is complex and numerous factors contribute to the observed vasoconstriction and vascular remodelling. In recent times, three main mechanistic pathways have been identified that are known to contribute to the development and progression of $\mathrm{PAH}$, namely the endothelin, nitric oxide and prostacyclin (prostaglandin $(\mathrm{PG}) \mathrm{I}_{2}$ ) pathways (fig. 1) [1].

Received: Aug 292014 | Accepted after revision: Oct 072014

Conflict of interest: Disclosures can be found alongside the online version of this article at err.ersjournals.com

Provenance: Publication of this peer-reviewed article was sponsored by Actelion Pharmaceuticals Ltd, Allschwil, Switzerland (principal sponsor, European Respiratory Review issue 134).

Copyright OERS 2014. ERR articles are open access and distributed under the terms of the Creative Commons Attribution Non-Commercial Licence 4.0. 
The endothelin pathway is a key mediator of pulmonary vascular remodelling through the induction of smooth muscle cell and fibroblast proliferation and vasoconstriction [2]. Activation of endothelin receptor type B also mediates vasodilation via the nitric oxide and prostacyclin pathways [2]. Activation of the nitric oxide signalling pathway leads to vasodilation and inhibits cell proliferation; phosphodiesterase type-5 (PDE-5) inhibitors augment this pathway by disrupting the catalysis of cGMP to GMP [3]. Finally, the $\mathrm{PGI}_{2}$ pathway has an established role in increasing vasodilation and inhibiting vascular smooth muscle cell proliferation and migration [4]. While numerous other players, including receptor tyrosine kinasemediated signalling and the serotonin receptor signalling, have been shown to play a role in the pathophysiology of the disease, to date, no therapies addressing these pathways have been approved.

In patients with $\mathrm{PAH}$, lower levels of endogenous $\mathrm{PGI}_{2}$ [5] and nitric oxide [6], and elevated levels of endothelin [7] have been observed. Due to the involvement and interaction of these three pathways in disease progression, using more than one class of drug (combination therapy) to target multiple disease pathways may potentially increase the overall impact on each or all of the mechanisms involved in PAH $[8,9]$ and, as a result, improve treatment success.

Although frequently used in clinical practice, supporting evidence for starting combination therapy in $\mathrm{PAH}$ patients, either sequentially (addition of a second treatment to initial monotherapy) or upfront (commencing with two or more therapies in treatment-naïve patients), was historically based on experience

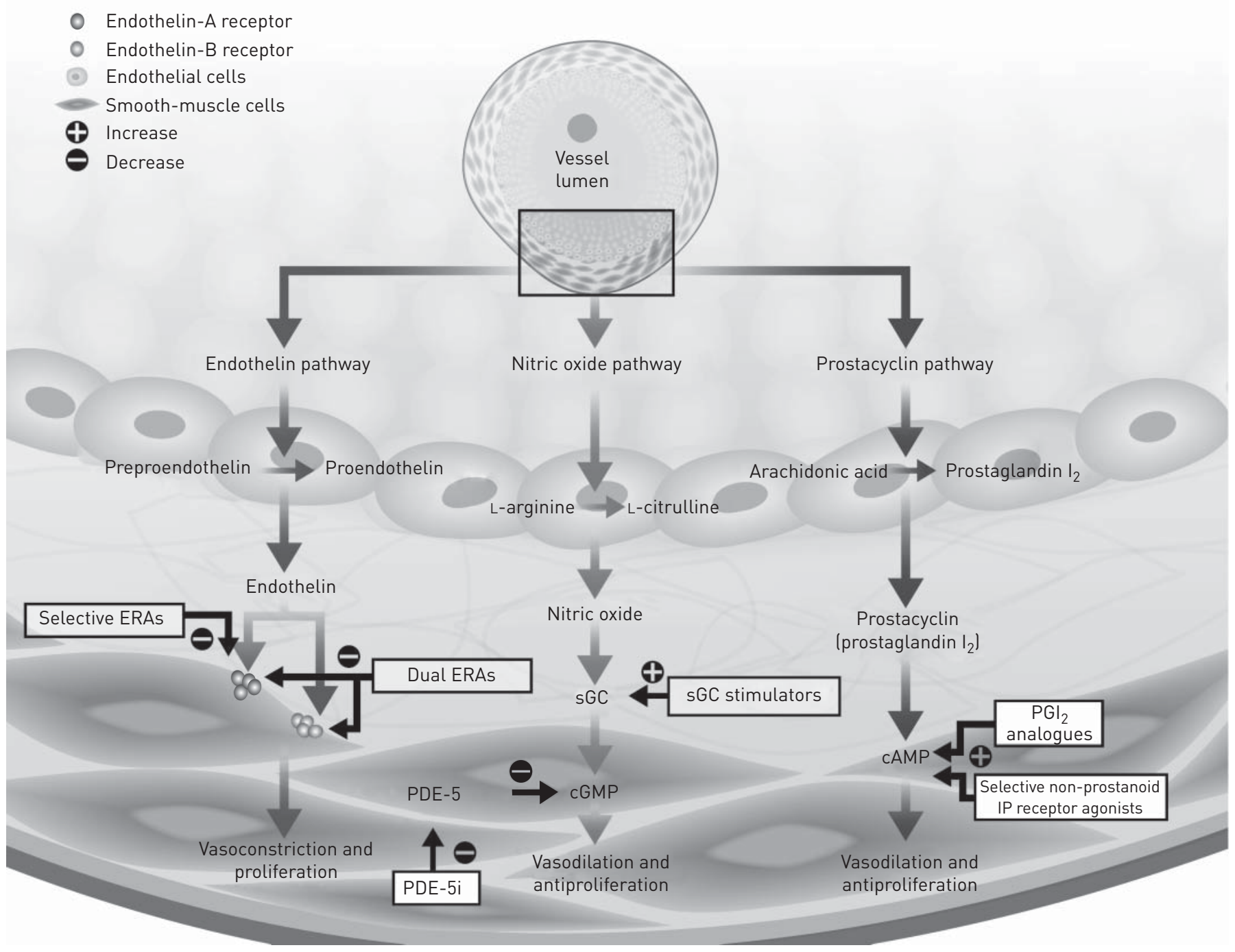

FIGURE 1 Schematic diagram of three biological pathways involved in the pathogenesis of pulmonary arterial hypertension. ERA: endothelin receptor antagonists; sGC: soluble guanine cyclase; PDE-5: phosphodiesterase type-5; PDE-5i: PDE-5 inhibitors; PGI ${ }_{2}$ : prostaglandin I $\mathrm{I}_{2}$ IP: I prostanoid. Reproduced and modified from [8] with permission from the publisher. 
from clinical practice and expert opinion, together with practicality and convenience, as supporting clinical trial data were lacking. In recent years, additional data from randomised controlled trials (RCTs) examining the effects of combination therapy have become available, accompanied by a shift towards evidence-based use of combination therapy in patients with PAH [10, 11]. In spite of this increasing evidence, observations from the US REVEAL (Registry to Evaluate Early and Long-term PAH Disease Management) registry have shown a lack of consistent use of combination therapy in patients with severe $\mathrm{PAH}$, with $34.9 \%$ of patients receiving only monotherapy at the time of death [12].

This article will review the rationale and evolving evidence for the use of both sequential and upfront combination therapies for $\mathrm{PAH}$, and discuss important treatment considerations.

\section{Current recommendations for the use of combination therapy in PAH}

PAH-targeted therapy is considered in symptomatic patients who are not vasoreactive or who are vasoreactive but display a suboptimal response to treatment with calcium channel blockers [10]. The current PAH treatment algorithm, as updated following the 5th World Symposium on Pulmonary Hypertension, recommends targeting at least one of the three main disease pathways (fig. 2) [11, 13]. The endothelin pathway is targeted by endothelin receptor antagonists such as bosentan, ambrisentan or macitentan, while the nitric oxide pathway is targeted through PDE-5 inhibitors that include sildenafil and tadalafil, and more recently, a soluble guanylate cyclase stimulator, riociguat [14]. Treatments targeting the prostacyclin pathway include epoprostenol, iloprost, treprostinil and beraprost [10].

The current treatment algorithm indicating the addition of a second treatment in addition to background therapy may be considered when an inadequate clinical response or deterioration is observed with monotherapy in patients in World Health Organization (WHO) functional class III/IV [10]. Since the 4th World Symposium on Pulmonary Hypertension recommendations in 2008, the level of evidence for sequential combination therapy has been upgraded to I-A in the 5th World Symposium on Pulmonary Hypertension recommendations in 2013 [9] following further data from RCTs and meta-analyses [15-20]. By contrast, evidence to support upfront combination therapy remains largely based on expert consensus and/or small studies, retrospective studies and registries. The 5th World Symposium on Pulmonary Hypertension has graded the evidence of initial combination therapy in WHO functional class III/IV as IIb-C [10].

Although guidelines provide some level of recommendation for sequential or upfront combination therapy, multiple combinations are possible between the approved PAH drugs. The efficacy and safety profile of each of these combinations may vary and it is, therefore, important to evaluate the available evidence for each individual combination when choosing an appropriate treatment regimen for a patient.

\section{Examining the current evidence for sequential combination therapy}

The inclusion of multiple background therapies in RCT design has significantly contributed to increasing the evidence for sequential combination therapies in PAH [14, 16-28].

In the past, as seen in table 1 , short-term RCTs have given conflicting results in patients with PAH. A metaanalysis of monotherapy compared with combination therapy in six RCTs (ranging from 12 to 16 weeks in

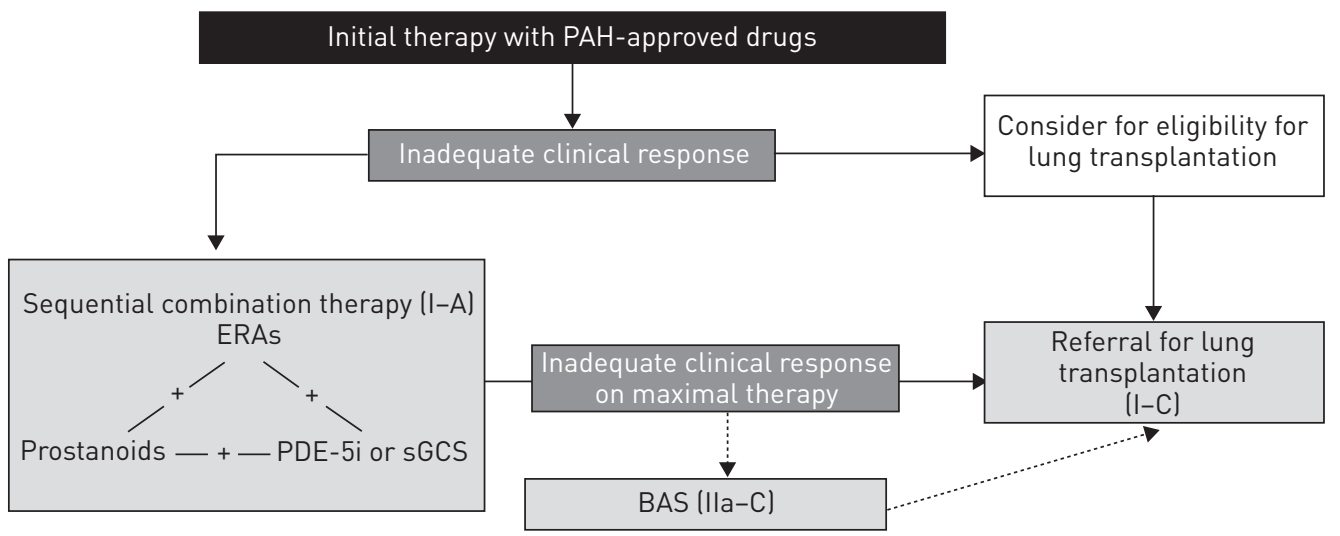

FIGURE 2 Treatment algorithm showing the class of recommendations and level of evidence for sequential combination therapy. PAH: pulmonary arterial hypertension; ERA: endothelin receptor antagonists; PDE-5i: phosphodiesterase type-5 inhibitor; sGCS: soluble guanine cyclase stimulator; BAS: balloon atrial septostomy. Reproduced from [10] with permission from the publisher. 
TABLE 1 Summary of randomised controlled trials of sequential combination therapy in pulmonary arterial hypertension

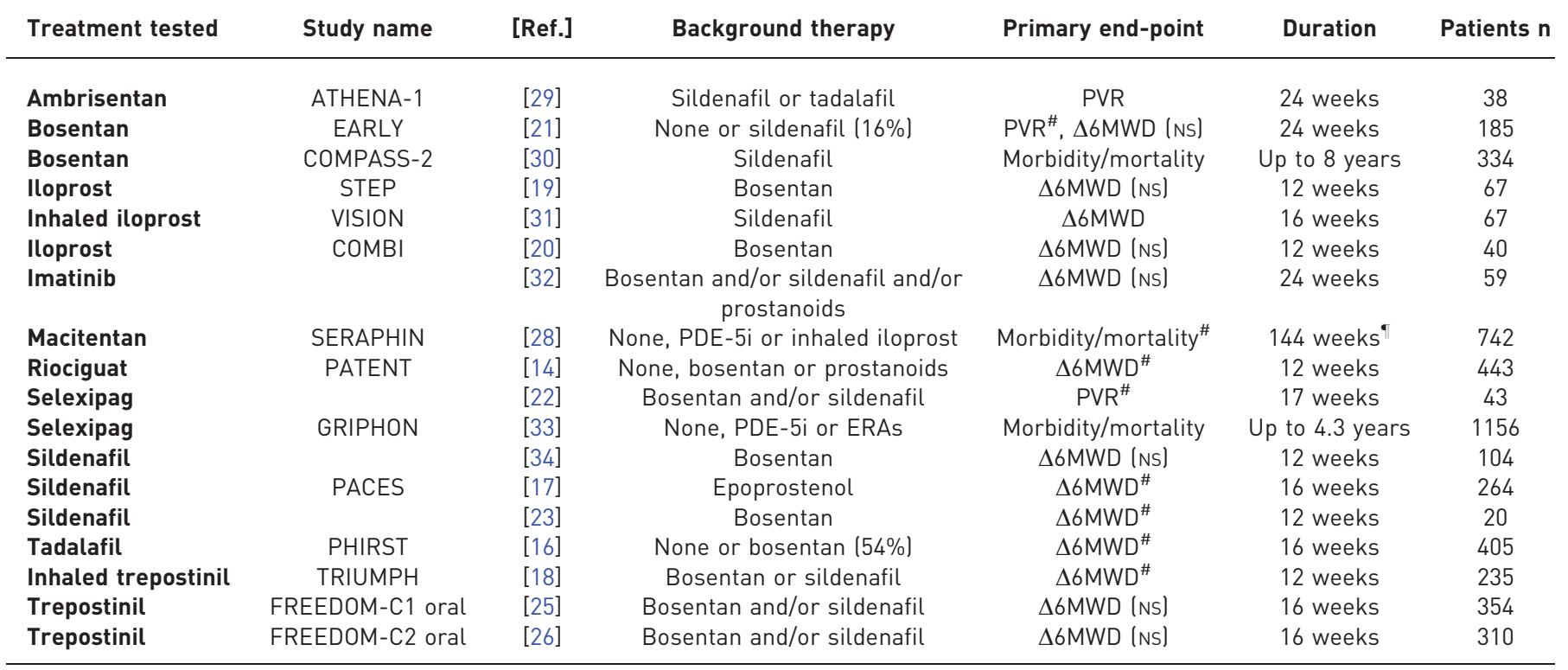

PVR: pulmonary vascular resistance; $\triangle 6 \mathrm{MWD}$ : change in 6-min walking distance; NS: nonsignificant; PDE-5i: phosphodiesterase type-5 inhibitor; ERAs: endothelin receptor antagonists. ": statistically significant; ": event-driven study. Data from [10].

duration) did not show a beneficial effect of sequential combination therapy on either a combined clinical worsening or survival end-point, although a modest improvement in exercise capacity was observed [15]. This finding was echoed by a systematic review and meta-analysis of 28 RCTs [35]. A separate meta-analysis of seven clinical trials indicated combination therapy was associated with beneficial effects in both exercise capacity and disease worsening, but no improvement was reported in survival compared to monotherapy [36].

A finding of nonsignificance for 6-min walking distance (6MWD) was reported for the 16-week FREEDOM-C1 and FREEDOM-C2 trials, both investigating oral trepostinil on a background of bosentan and/or sildenafil $[25,26]$. In addition, a 12-week study of sildenafil in addition to background bosentan therapy did not show a beneficial effect on 6MWD [34]. However, other recent results have shown beneficial effects of combination therapy versus monotherapy alone. The 12-week PATENT-1 study showed that riociguat improved $6 \mathrm{MWD}$ compared to placebo in patients receiving riociguat in addition to endothelin receptor antagonists or prostanoids [14]. The COMPASS-2 trial was a long-term study of the use of bosentan in addition to background sildenafil. While the study did not meet its primary end-point of reducing time to first morbidity or mortality event, an exploratory analysis indicated that bosentan in addition to sildenafil led to an improvement in 6MWD at week 16 [30].

The effect of PAH therapies on long-term outcomes of PAH patients has now been evaluated in large RCTs assessing the risk of morbidity/mortality events over time as a primary outcome. A subgroup analysis from the SERAPHIN study $(\mathrm{n}=317$; median treatment duration 2.2 years) showed that the addition of macitentan significantly reduced the risk of a composite morbidity/mortality event by $38 \%(p=0.009)$ in patients on stable background therapies for PAH; a PDE-5 inhibitor (predominantly sildenafil) was used in $96 \%$ of these patients (fig. 3) [28]. In contrast, COMPASS-2 did not meet the primary end-point of time to first morbidity/mortality event, with a risk reduction of $17 \%$ with bosentan versus placebo on background sildenafil treatment $(\mathrm{p}=0.25)[30]$.

\section{The use of upfront combination therapy}

Compared to sequential combination therapy, much less is known about the benefits of upfront combination therapy; information comes from trials mainly evaluating drugs in severe PAH patients [27, 37]. In the BREATHE-2 trial, a double-blind, placebo-controlled prospective study $(n=33)$, the efficacy and safety of first-line combined bosentan plus epoprostenol versus epoprostenol alone was investigated in severe PAH patients [27]. Haemodynamic measurements, exercise capacity and functional capacity showed improvements in both treatment groups at week 16, with a trend towards a greater improvement in all these parameters in the combined treatment group (although none achieved statistical significance compared to monotherapy) [27]. An observational study $(n=23)$ reported long-term $(\sim 30$ months $)$ improvements in 6 MWD and pulmonary 


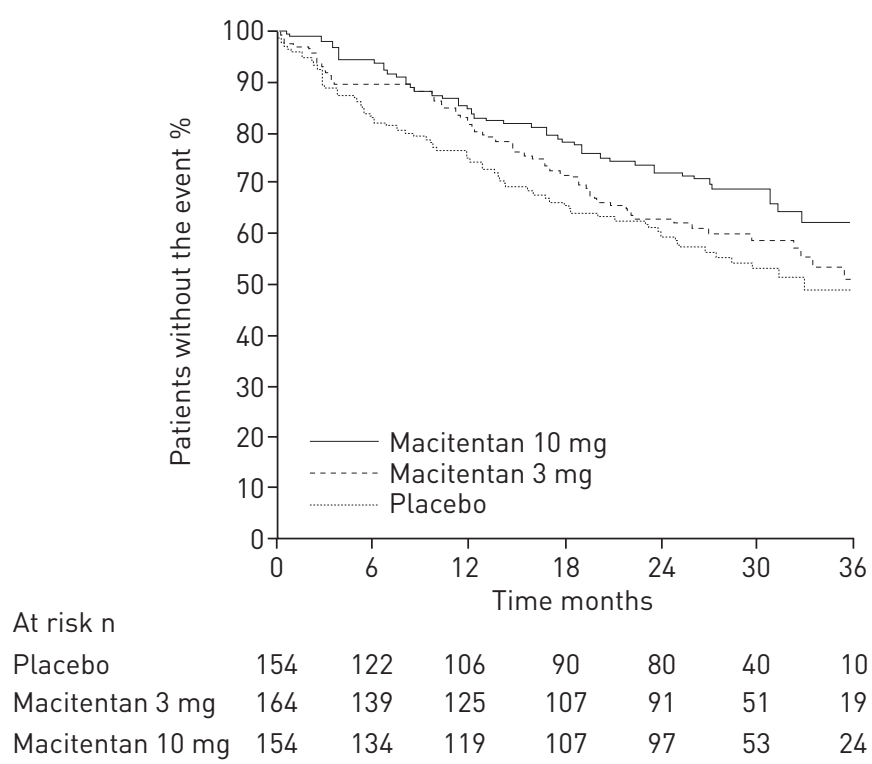

FIGURE 3 Kaplan-Meier estimates for effect of macitentan on morbidity or mortality as a first event in patients with background pulmonary arterial hypertension therapy at baseline. Reproduced from [28] with permission from the publisher.

vascular resistance in patients receiving upfront combination therapy with bosentan and intravenous epoprostanol, compared to i.v. epoprostanol monotherapy, with a trend $(\mathrm{p}=0.07)$ towards improvement in overall survival [38]. More recently, results from a retrospective analysis of 77 patient records in Sweden indicated that upfront combination therapy $(n=13)$ was associated with a reduction in haemodynamics compared to monotherapy, but no statistical significance in 1-, 2- or 5-year survival rate [39].

Results from the long-term, randomised, multicentre AMBITION trial, which assessed the upfront combination of ambrisentan and tadalafil compared with each treatment given as monotherapy in patients with PAH, are expected to contribute to the current understanding of this treatment approach [40]. Further studies are required to evaluate differences in long-term outcomes and ascertain whether the benefits of upfront combination therapy merit expanded clinical use.

Triple upfront combination therapy has been proposed as an alternative treatment approach in patients with severe PAH. A prospective, observational analysis of 19 patients with idiopathic or heritable PAH has shown significant improvements compared with baseline in haemodynamics, WHO functional class status and 6MWD using triple combination therapy with i.v. epoprostenol, bosentan and sildenafil [37]. Of particular note was the 3-year survival rate of $100 \%$, paired with achievement of WHO functional class I or II in all patients, in a patient population with severe PAH that would otherwise have an expected 3-year survival of $49 \%$ [37]. These results must be interpreted with care due to the small patient numbers and nonrandomised study design. However, the promising results observed by targeting all three disease pathways in combination, in patients with severe disease, could be explained as a result of action on all three distinct yet overlapping causal pathways, or through ensuring that whichever specific pathway is the predominant causal mechanism in a specific patient is targeted from the outset [9].

\section{Additional considerations for sequential versus upfront combination therapy}

As evidence for sequential combination therapy increases, it may begin to impact on the choice of first-line monotherapy. Where possible, consideration should be given to the suitability of a particular monotherapy for successful future combination therapy in the patient concerned. Contrary to the impression generated by a general IA recommendation for the use of sequential combination therapy according to the 5th World Symposium on Pulmonary Hypertension, the evidence for particular constellations is very different and specific drug combinations should be preferred over others.

Patient age and physical status can also be an important factor in treatment. Treatment can be re-evaluated as disease progresses, and as patients move between WHO functional classes, with younger, healthier patients potentially being considered for earlier, harder-hitting regimens in order to achieve early and sustainable improvement. The difficulties of improving exercise capacity in patients already on monotherapy (the ceiling effect) is well established, and this is one of the many reasons that could explain 
the inconsistency of results across the different clinical trials shown in table 1. Furthermore, it is important to note that some treatment combinations are contraindicated, specifically riociguat and PDE-5 inhibitors.

Finally, when initiating combination therapy, one must also consider the transition from therapy to transplantation, as patients receiving combination treatments are likely to be characterised by more progressive disease status [41]. As any delays in referral can be compounded by lack of donor organ availability and are likely to result in clinical worsening in patients requiring transplantation, it is recommended that a patient's eligibility for transplant be assessed in parallel to initiation of sequential combination therapy.

\section{Conclusion}

The evidence provided by longer term RCTs indicates that specific combination therapies for PAH may provide an effective therapeutic approach for patients. Combination therapy is now considered an important part of the treatment algorithm for PAH. In future, this may impact the physician's decision on first-line therapy, as certain combinations are preferred. It will be important for physicians to evaluate carefully the growing body of evidence for combination therapy in order to provide their patients with the treatment plan that will result in the best possible outcome.

\section{Acknowledgements}

Medical writing support was provided by Nason Ma'ani (apothecom scopemedical ltd, Sevenoaks, UK), which was funded by Actelion Pharmaceuticals Ltd (Allschwil, Switzerland).

\section{References}

1 Humbert M, Morrell NW, Archer SL, et al. Cellular and molecular pathobiology of pulmonary arterial hypertension. J Am Coll Cardiol 2004; 43: Suppl. 12, 13S-24S

2 Sitbon O, Morrell N. Pathways in pulmonary arterial hypertension: the future is here. Eur Respir Rev 2012; 21: 321-327.

3 Chen CN, Watson G, Zhao L. Cyclic guanosine monophosphate signalling pathway in pulmonary arterial hypertension. Vascul Pharmacol 2013; 58: 211-218.

4 Mubarak KK. A review of prostaglandin analogs in the management of patients with pulmonary arterial hypertension. Respir Med 2010; 104: 9-21.

5 Christman BW, McPherson CD, Newman JH, et al. An imbalance between the excretion of thromboxane and prostacyclin metabolites in pulmonary hypertension. N Engl J Med 1992; 327: 70-75.

6 Giaid A, Saleh D. Reduced expression of endothelial nitric oxide synthase in the lungs of patients with pulmonary hypertension. N Engl J Med 1995; 333: 214-221.

7 Giaid A, Yanagisawa M, Langleben D, et al. Expression of endothelin-1 in the lungs of patients with pulmonary hypertension. N Engl J Med 1993; 328: 1732-1739.

8 Humbert M, Sitbon O, Simonneau G. Treatment of pulmonary arterial hypertension. N Engl J Med 2004; 351 : $1425-1436$.

9 McGoon MD. Upfront triple therapy for pulmonary arterial hypertension: is three a crowd or critical mass? Eur Respir J 2014; 43: 1556-1559.

10 Galiè N, Corris PA, Frost A, et al. Updated treatment algorithm of pulmonary arterial hypertension. J Am Coll Cardiol 2013; 62: Suppl. 25, D60-D72

11 Galiè N, Hoeper MM, Humbert M, et al. Guidelines for the diagnosis and treatment of pulmonary hypertension: the Task Force for the Diagnosis and Treatment of Pulmonary Hypertension of the European Society of Cardiology (ESC) and the European Respiratory Society (ERS), endorsed by the International Society of Heart and Lung Transplantation (ISHLT). Eur Heart J 2009; 30: 2493-2537.

12 Farber HW, Miller DP, Meltzer LA, et al. Treatment of patients with pulmonary arterial hypertension at the time of death or deterioration to functional class IV: insights from the REVEAL Registry. J Heart Lung Transplant 2013; 32: $1114-1122$.

13 McLaughlin VV, Archer SL, Badesch DB, et al. ACCF/AHA 2009 expert consensus document on pulmonary hypertension a report of the American College of Cardiology Foundation Task Force on Expert Consensus Documents and the American Heart Association developed in collaboration with the American College of Chest Physicians; American Thoracic Society, Inc.; and the Pulmonary Hypertension Association. J Am Coll Cardiol 2009; 53: 1573-1619.

14 Ghofrani HA, Galiè N, Grimminger F, et al. Riociguat for the treatment of pulmonary arterial hypertension. N Engl J Med 2013; 369: 330-340.

15 Fox BD, Shimony A, Langleben D. Meta-analysis of monotherapy versus combination therapy for pulmonary arterial hypertension. Am J Cardiol 2011; 108: 1177-1182.

16 Galiè N, Brundage BH, Ghofrani HA, et al. Tadalafil therapy for pulmonary arterial hypertension. Circulation 2009; 119: 2894-2903.

17 Simonneau G, Rubin LJ, Galiè N, et al. Addition of sildenafil to long-term intravenous epoprostenol therapy in patients with pulmonary arterial hypertension: a randomized trial. Ann Intern Med 2008; 149: 521-530.

18 McLaughlin VV, Benza RL, Rubin LJ, et al. Addition of inhaled treprostinil to oral therapy for pulmonary arterial hypertension: a randomized controlled clinical trial. J Am Coll Cardiol 2010; 55: 1915-1922.

19 McLaughlin VV, Oudiz RJ, Frost A, et al. Randomized study of adding inhaled iloprost to existing bosentan in pulmonary arterial hypertension. Am J Respir Crit Care Med 2006; 174: 1257-1263.

20 Hoeper MM, Leuchte H, Halank M, et al. Combining inhaled iloprost with bosentan in patients with idiopathic pulmonary arterial hypertension. Eur Respir J 2006; 28: 691-694. 
Galiè N, Rubin L, Hoeper M, et al. Treatment of patients with mildly symptomatic pulmonary arterial hypertension with bosentan (EARLY study): a double-blind, randomised controlled trial. Lancet 2008; 371: $2093-2100$.

22 Simonneau G, Torbicki A, Hoeper MM, et al. Selexipag: an oral, selective prostacyclin receptor agonist for the treatment of pulmonary arterial hypertension. Eur Respir J 2012; 40: 874-880.

23 Iversen K, Jensen AS, Jensen TV, et al. Combination therapy with bosentan and sildenafil in Eisenmenger syndrome: a randomized, placebo-controlled, double-blinded trial. Eur Heart J 2010; 31: 1124-1131.

24 Galiè N, Negro L, Simonneau G. The use of combination therapy in pulmonary arterial hypertension: new developments. Eur Respir Rev 2009; 18: 148-153.

25 Tapson VF, Torres F, Kermeen F, et al. Oral treprostinil for the treatment of pulmonary arterial hypertension in patients on background endothelin receptor antagonist and/or phosphodiesterase type 5 inhibitor therapy (the FREEDOM-C study): a randomized controlled trial. Chest 2012; 142: 1383-1390.

26 Tapson VF, Jing ZC, Xu KF, et al. Oral treprostinil for the treatment of pulmonary arterial hypertension in patients receiving background endothelin receptor antagonist and phosphodiesterase type 5 inhibitor therapy (the FREEDOM-C2 study): a randomized controlled trial. Chest 2013; 144: 952-958.

27 Humbert M, Barst RJ, Robbins IM, et al. Combination of bosentan with epoprostenol in pulmonary arterial hypertension: BREATHE-2. Eur Respir J 2004; 24: 353-359.

28 Pulido T, Adzerikho I, Channick RN, et al. Macitentan and morbidity and mortality in pulmonary arterial hypertension. N Engl J Med 2013; 369: 809-818.

29 Clinicaltrials.gov. Study of add-on ambrisentan therapy to background phosphodiesterase type-5 inhibitor (PDE5i) therapy in pulmonary arterial hypertension (ATHENA-1). NCT00617305. https://clinicaltrials.gov/ct2/show/ NCT00617305?term = NCT00617305\&rank=1 Date last updated: June 22, 2012. Date last accessed: September 30, 2014

30 McLaughlin V, Channick R, Ghofrani HA, et al. Effect of bosentan and sildenafil combination therapy on morbidity and mortality in pulmonary arterial hypertensino (PAH): results from the COMPASS-2 study. Chest 2014; 146: 860A.

31 Clinicaltrials.gov. The "VISION" Trial: ventavis inhalation with sildenafil to improve and optimize pulmonary arterial hypertension. NCT00302211. https://clinicaltrials.gov/ct2/show/NCT00302211?term = NCT00302211\&rank=1 Date last updated: May 12, 2011. Date last accessed: September 30, 2014.

32 Ghofrani HA, Morrell NW, Hoeper MM, et al. Imatinib in pulmonary arterial hypertension patients with inadequate response to established therapy. Am J Respir Crit Care Med 2010; 182: 1171-1177.

33 Clinicaltrials.gov. Selexipag (ACT-293987) in pulmonary arterial hypertension, GRIPHON Trial. NCT01106014. http://clinicaltrials.gov/ct2/show/NCT01106014?term=griphon\&rank=1 Date last updated: September 25, 2014. Date last accessed: September 30, 2014.

34 Clincaltrials.gov. Assess the efficacy and safety of sildenafil when added to bosentan in the treatment of pulmonary arterial hypertension. NCT00323297. https://clinicaltrials.gov/ct2/show/NCT00323297?term=NCT00323297\&rank=1 Date last updated: July 17, 2014. Date last accessed: September 30, 2014.

35 Coeytaux RR, Schmit KM, Kraft BD, et al. Comparative effectiveness and safety of drug therapy for pulmonary arterial hypertension: a systematic review and meta-analysis. Chest 2014; 145: 1055-1063.

36 Zhu B, Wang L, Sun L, et al. Combination therapy improves exercise capacity and reduces risk of clinical worsening in patients with pulmonary arterial hypertension: a meta-analysis. J Cardiovasc Pharmacol 2012; 60: 342-346.

37 Sitbon O, Jaïs X, Savale L, et al. Upfront triple combination therapy in pulmonary arterial hypertension: a pilot study. Eur Respir J 2014; 43: 1691-1697.

38 Kemp K, Savale L, O'Callaghan DS, et al. Usefulness of first-line combination therapy with epoprostenol and bosentan in pulmonary arterial hypertension: an observational study. J Heart Lung Transplant 2012; 31: 150-158.

39 Kylhammar D, Persson L, Hesselstrand R, et al. Prognosis and response to first-line single and combination therapy in pulmonary arterial hypertension. Scand Cardiovasc J 2014; 48: 223-233.

40 Clinicaltrials.gov. A study of first-line ambrisentan and tadalafil combination therapy in subjects with pulmonary arterial hypertension (PAH) (AMBITION). NCT01178073. http://clinicaltrials.gov/ct2/show/NCT01178073 Date last updated: October 2, 2014. Date last accessed: September 30, 2014.

41 Corris P, Degano B. Severe pulmonary arterial hypertension: treatment options and the bridge to transplant. Eur Respir Rev 2014; 23: 488-497. 